\title{
Characterization of the angular response of a multi-directional spectroradiometer for measuring spectral radiance
}

\author{
Mario Tobar Foster* (D), Eduardo Luiz Weide, Angelika Niedzwiedz, Jens Duffert and Gunther Seckmeyer
}

\section{*Correspondence:}

tobar-foster@muk.uni-hannover.de

Institute of Meteorology and

Climatology, Leibniz University

Hannover, D-30419, Hannover, Germany

\begin{abstract}
Despite its importance, few instruments are able to measure the angular distribution of the solar spectrum with a high spectral and temporal resolution. We present a novel characterization method of the multi-directional entrance optics of the AMUDIS (Advanced MUltiDlrectional Spectroradiometer) which is a multidirectional spectroradiometer based on three CCD image sensors combined with imaging spectrographs. The new type of entrance optics consists of 435 different optical fibres uniformly distributed along 145 directions covering the upper hemisphere and allowing simultaneous measurements of the radiance in the ultraviolet, visible and near infrared part of the spectrum, ranging from $280 \mathrm{~nm}$ to $1700 \mathrm{~nm}$. The experimental setup for characterizing the multidirectional entrance optics is based on a $100 \mathrm{~W}$ halogen lamp and a robotic arm, which moves the lamp tangentially over the surface of a virtual sphere of $102.5 \mathrm{~cm}$ radius around the entrance optics. The characterization revealed misalignments in the position of the optical fibres of up to $3^{\circ}$ (which can affect radiance measurements, specially under broken clouds conditions). The novel characterization method improved 3-fold the alignment up to $\pm 0.1^{\circ}$
\end{abstract}

Keywords: Solar radiation, Spectroradiometry, Spectral radiance

\section{Introduction}

The spectrum of solar irradiance at the ground has been measured from the ultraviolet (UV) to the near infrared (NIR) since a long time [1]. While previous efforts have been mostly concentrated on the measurement of spectral irradiance, less emphasis has been given to ground-based measurements of spectral radiance [2-5]. The main reason is the limited quantity of instruments capable of measuring the radiance distribution with a high spectral and temporal resolution.

Most of the existing instruments for measuring the radiance distribution are based on scanning the sky with a 2-axis tracker, which leads to a low temporal resolution of minutes at best $[2-4,6,7]$. For instance, a scan around local noon, with about 100 points evenly distributed on the west or east side of the hemisphere, takes 8 minutes per wavelength [8]. This shortcoming is of particular concern under cloudy-sky conditions within

(c) The Author(s). licensee Springer on behalf of EPJ. 2021, corrected publication 2022 Open Access This article is licensed under a Creative Commons Attribution 4.0 International License, which permits use, sharing, adaptation, distribution and reproduction in any medium or format, as long as you give appropriate credit to the original author(s) and the source, provide a link to the Creative Commons licence, and indicate if changes were made. The images or other third party material in this article are included in the article's Creative Commons licence, unless indicated otherwise in a credit line to the material. If material is not included in the article's Creative Commons licence and your intended use is not permitted by statutory regulation or exceeds the permitted use, you will need to obtain permission directly from the copyright holder. To view a copy of this licence, visit http://creativecommons.org/ licenses/by/4.0/. 
which short-term changes in the solar radiation are likely [9]. A more complete instrument called PARABOLA [10-12], capable of measuring radiance in a wide spectral range $(350 \mathrm{~nm}$ to $1650 \mathrm{~nm})$ and in a relatively short time (about 10 seconds), which can measure both in the upper and lower hemisphere, can only record radiation at certain wavelengths $(444,551,650,860,944,1028$ and $1650 \mathrm{~nm})$ or at most in a continuous range of $300 \mathrm{~nm}$ in the visible spectral range $(400-700 \mathrm{~nm}$ centred at $581 \mathrm{~nm})$. Spectroradiometers that scan the sky require (too) long measuring times. During the measuring time the sky is changing which leads to artefacts in the results. With such scanning instruments you need either constant conditions (which are limited in their occurrence) or long averaging times, which uncover relevant information. An approach with simultaneous measurements of sky radiance can overcome this difficulty.

Although method based on Hemispherical Sky Images (HSI) are an alternative for nearly instantaneous measurements of the radiance distribution, HSI cameras only cover the visible (VIS) range, operating in three wavelength bands peaking at $464 \mathrm{~nm}, 534 \mathrm{~nm}$ and $626 \mathrm{~nm}[13,14]$. Another technology for spectral radiance measurements (called 4D Imager) has been recently developed by Zuber et al., 2017 [15]. It is based on snapshot imaging, a line-scanning spectrometer and a facet mirror. The prototype of this technology has a high spatial resolution (196 channels) but a relatively low spectral range (adjustable $500 \mathrm{~nm}$ range) and a relatively low spectral resolution of about $10 \mathrm{~nm}$ only. Although the mirror approach of the $4 \mathrm{D}$ imager is a promising, there are unsolved problems concerning the input optics which does not cover the whole sky. Therefore a solution with fibre optics is shown to be better suited for the time being.

As an attempt to solve the shortcomings of existing technologies, a multi-directional spectroradiometer (MUDIS) [5] was developed at the Institute of Meteorology and Climatology of the Leibniz University of Hannover, Germany. MUDIS allows carrying out measurements of fast-changing radiance distribution within seconds. It is based on a UV-sensitive CCD camera, an Offner imaging spectroradiometer, and multi-directional entrance optics, consisting of 113 fibres. The MUDIS was scaled up to the AMUDIS (Advanced MUlti-Directional Spectroradiometer) [16] with a higher spectral and spatial resolution and an extended spectral range allowing spectral measurements from $280 \mathrm{~nm}$ to $1700 \mathrm{~nm}$ and a spectral resolution of $1 \mathrm{~nm}$. AMUDIS is capable to measure the sky radiance with a temporal resolution of seconds, opening new possibilities for the study of temporary radiance changes, which is important in many fields, like the biological impact of UV radiation [17], solar energy production, for the calculation of radiation quantities such as e.g. the spectral radiance, which can be used for further calculation of radiative transfer equation, erythema and vitamin D efficacy, fast changes of the sky radiance distribution due to the influence of clouds as a couple of examples.

Similar to the MUDIS the AMUDIS is fitted with a Multi-Directional Input Optics (MDIO), which is what we have here characterized. MDIO is a hemispherical-shaped aluminium dome with 435 optical fibres evenly distributed in 145 different positions in the azimuth and zenith angles (Fig. 1).

Three optical fibres, one for each spectral region (UV, VIS and NIR) with a diameter of $50 \mu \mathrm{m}$ are set at each of the 145 apertures of the dome. The optical fibres are aligned along a slit in three metallic cylinders of $20 \mathrm{~mm}$ diameter and $50 \mathrm{~mm}$ length. Each of them is connected to the entrance of the corresponding spectroradiometer. The channels of the MDIO are shown in the Fig. 2. 


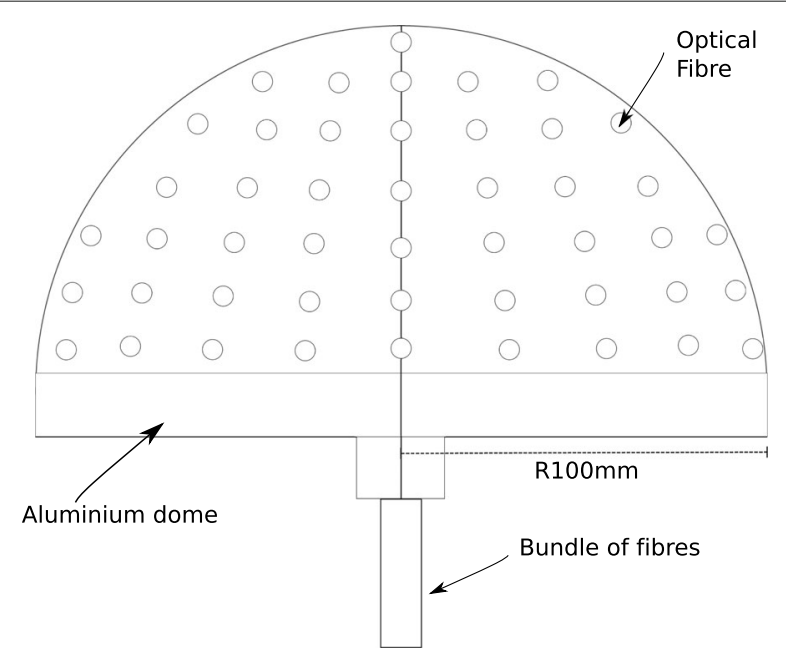

Fig. 1 Scheme of the multi-directional entrance optics (MDIO) of AMUDIS system. 435 optical fibres are distributed on the dome pointing at 145 different directions of the sky. At the end of the optical fibre bundle. the fibres are vertically aligned on a slit which can be connected to the spectroradiometer

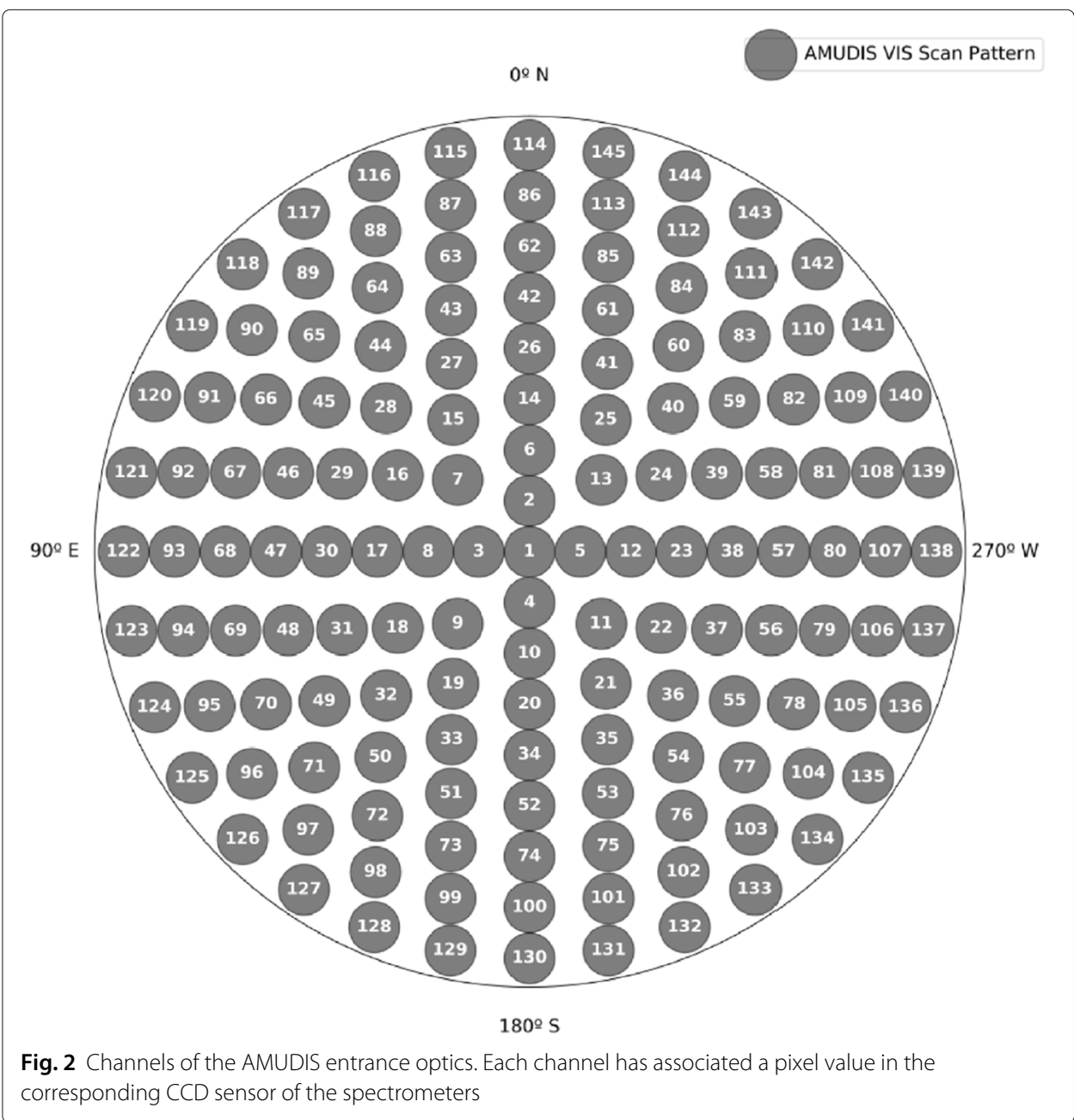


Due to the fact that the entrance optics cannot be perfectly aligned during the production process and that the fibres may not be cut in exactly the same way, both the optical fibre position and the cutting edge of the individual fibre on the dome may show misalignment. Therefore, a full characterization of the MDIO's angular response is necessary.

The disadvantage of the fibre approach is the sensitivity of the transmission of these thin fibre due to bending of the fibres. This leads to the need to develop new calibration methods [18] and to the need to calibrate the instrument at the location of deployment. Furthermore, the FOV depends on the material processing of the fibre at the opening. Irregularities in it can lead to a shadow throw in the FOV of the fibre and reduction of the signal or/and asymmetries in the FOV. The material-dependent ageing process of the fibres must also be considered.

\section{Measuring the angular response}

\section{Angular response of a unidirectional radiance input optics}

The angular response of a spectroradiometer can be measured by moving a lamp around the radiometer and measuring the spectrometer's signal as a function of the angle of incidence [19]. The experimental setup used for a unidirectional radiance entrance optics is similar to described by Knoop et al., 2018 [20]: the radiance entrance optics is mounted on a rotating table with $(0.25 \pm 0.06)^{\circ}$ accuracy, which can be manually rotated in the azimuthal plane. The input optics aperture was aligned with the vertical rotation axis of the rotating table (Fig. 3). A $100 \mathrm{~W}$ low-voltage halogen lamp without reflector (OSRAM model 64623 HLX) is mounted on the optical table and aligned vertically and horizontally by using a laser. A stable power supply with a current output of $8 \mathrm{~A}$ was used to maintain constant irradiance emitted by the lamp and reduce the uncertainties of the measurement [21]. The radiance is measured for different lamp positions as a function of the angle $\theta$, using angular steps of $0.5^{\circ}$.

\section{Angular response of a multi-directional entrance optics (MDIO)}

Each optical fibre of the MDIO was considered an independent unidirectional radiance entrance optics. We applied the same method described above but we extended it from a 2-dimensional plane to a 3-dimensional space by moving the lamp over the surface of

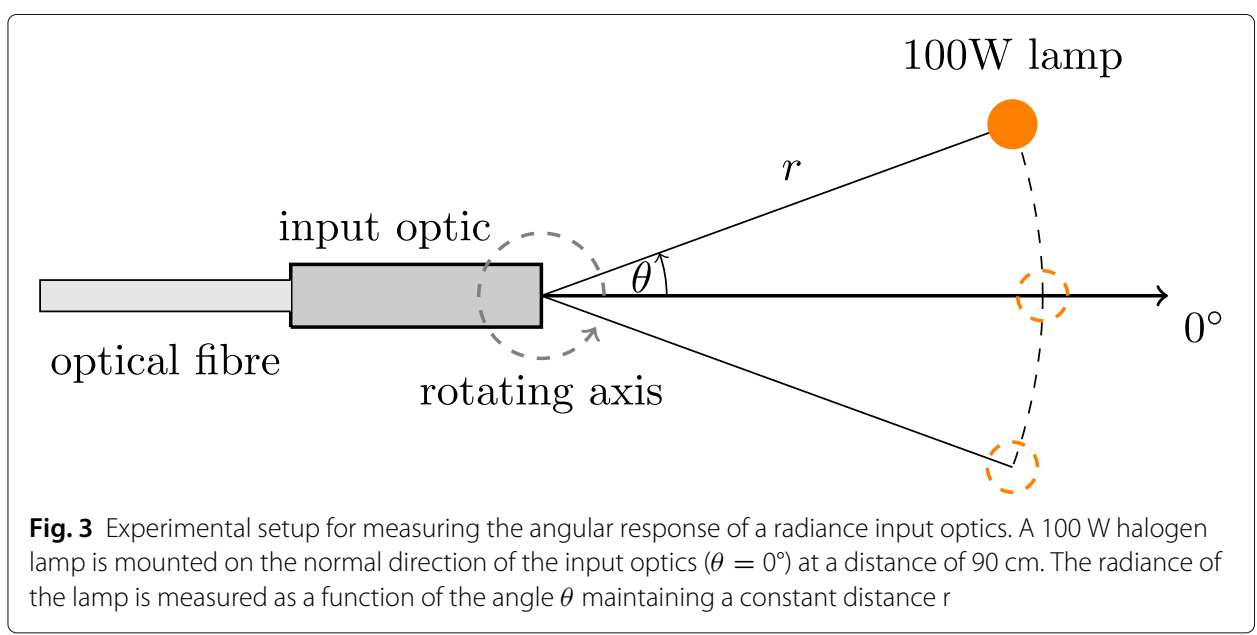


a virtual sphere of radius r. (Fig. 4). For that purpose, a 6-axis robotic arm [22] with a working radius of $1300 \mathrm{~mm}$ was used.

Due to its length, the robotic arm cannot cover the whole virtual sphere. For that reason, the robot was mounted on an $80 \times 40 \mathrm{~mm}$ aluminium-profile structure with three $80 \times 80$ mm mounting points (see Fig. 5). Using the first mounting point (Position 1 ) the robot reached zenith angles between $0^{\circ}$ and $36^{\circ}$ and azimuths of $\pm 22.5^{\circ}$ with respect to the input optics centre. The second mounting point (Position 2) covered zenith angles between $36^{\circ}$ and $60^{\circ}$. A third mounting point (Position 3) was used to cover the remaining angles between $60^{\circ}$ and $90^{\circ}$ in the zenithal direction. In total, we divided the scanned area into 28 sections ( $2 \times 8$ and $1 \times 12$ sections). Since the movements can affect the responsivity of the optical fibres, both the spectroradiometer and the entrance optics were mounted on the same rotating table of $40 \mathrm{~cm}$ diameter.

A total of 54,400 positions were required for scanning the virtual sphere at an angular resolution of $1^{\circ}$. Therefore, an automation of the measuring system was necessary; an Arduino UNO (a microcontroller board based on the ATmega328P) was used. It was also implemented as a bridge between the AMUDIS software and the robot software. The script enables a sequence of movements in which the robotic arm moves the lamp to the first position to scan over the virtual sphere. Once reaching its first target position, the robot sends a pulse signal to the AMUDIS software through an I/O output. AMUDIS receives this signal and makes a measurement of the spectral radiance. When the first measurement is finished, the AMUDIS software sends another signal to the

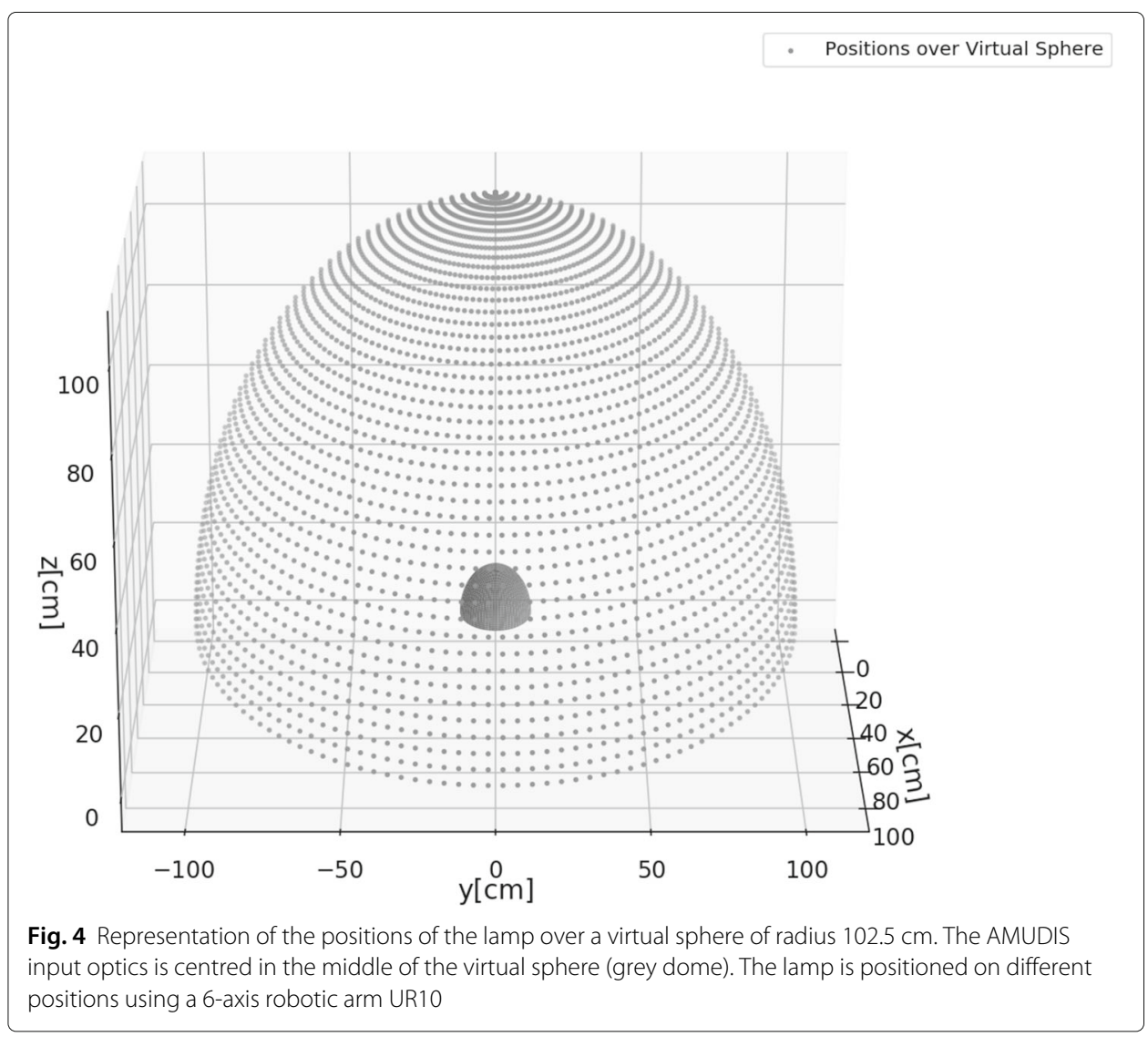




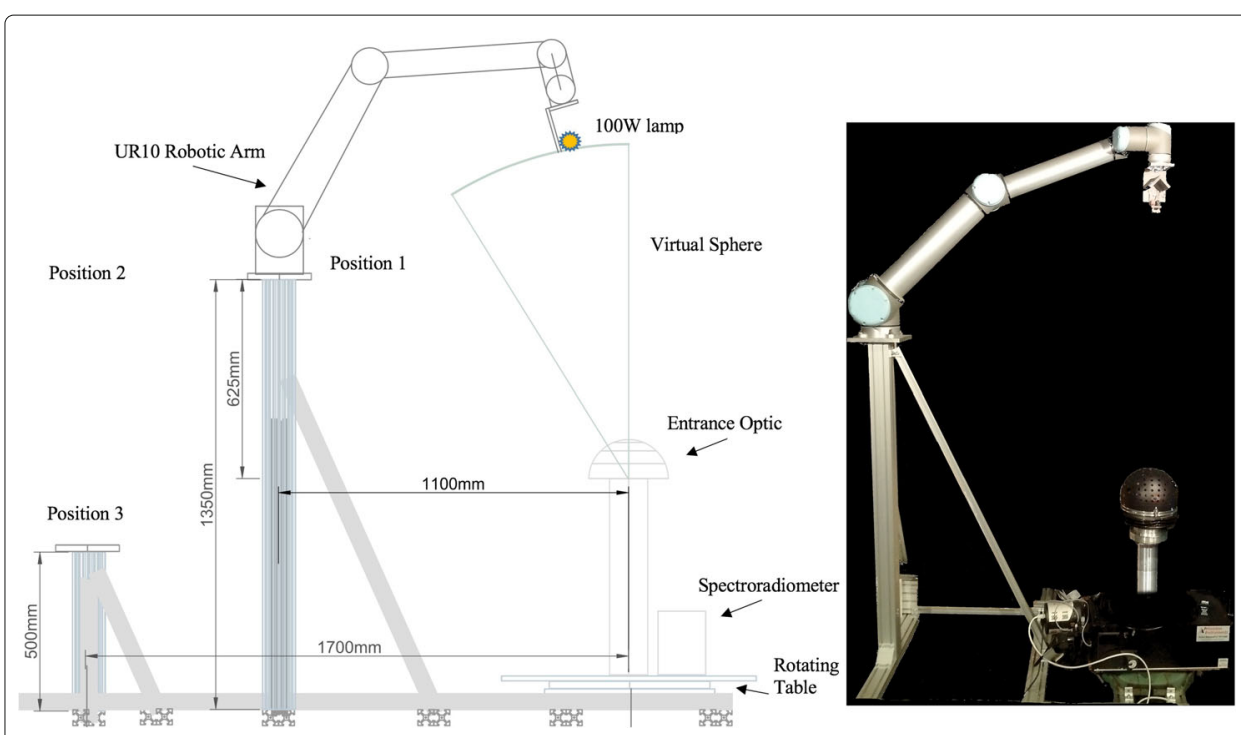

Fig. 5 Experimental setup for the angular response measurements of a multi-directional input optics. Three mounting points are used for the same robot, Position 1 for the upper area between $0^{\circ}$ and $36^{\circ}$, Position 2 (using the same aluminium-profile as Position 1 but fixed on Position 3 base) for the section between $36^{\circ}$ and $60^{\circ}$, and Position 3 for the lower area between $60^{\circ}$ to $90^{\circ}$. A rotation table is used to measure the radiance in different azimuthal sections

robot, such that the robotic arm moves the lamp to the next target position over the virtual hemisphere and the process continues up to the last position defined in the script.

\section{Optimization of the method}

The spherical-coordinate system used by the robot for the calculation of the lamp position over the virtual sphere causes the spatial distance between points near to the zenith angle to tend towards zero (Fig. 6a) for example, the spatial distance between a point positioned at $1^{\circ}$ zenith and separated $1^{\circ}$ in the azimuth is $3.12 \mathrm{~mm}$, that compared to a point positioned at $20^{\circ}$ zenith and the same azimuth separation is $61 \mathrm{~mm}$, it is only a $4.9 \%$ of the first spatial distance, resulting in a high number of obsolete points for the characterization. To avoid extensive measurement times, a correction method for the angular position was applied. First at all, a spatial distance $s$ over the virtual sphere of radius $R$ on the azimuth plane (zenith angle equal to $90^{\circ}$ ) was defined. The $\operatorname{arc} s$ for a zenith angle $\theta_{z}$ of $90^{\circ}$ was approximated as $R \cdot \sin \alpha_{p}$ (when $\alpha_{p}$ is small), where $\alpha_{p}$ is the azimuth angle.

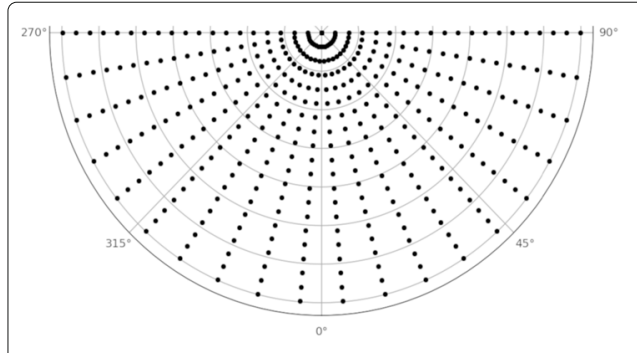

a)

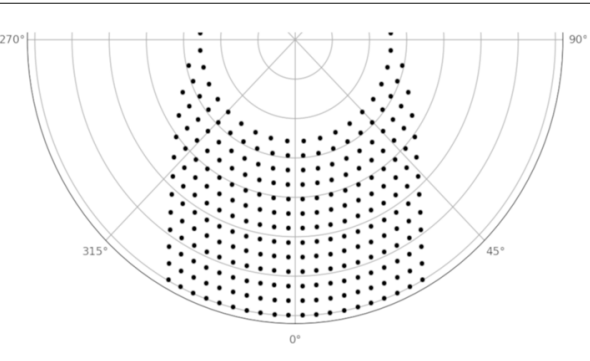

b)

Fig. 6 Polar projection of the position of the lamp over the surface of the virtual sphere. a) Positions without angular correction, which implies that the distance between points change as a function of zenith angle. b) corrected position using equation 1, which allows for a constant distance between points 
Hence, a corrected angle $\alpha_{c}$ as a function of the zenith angle $\theta_{z}$ is calculated by Equation 1 .

$$
\alpha_{c}\left(\theta_{z}\right)=\alpha_{p} \cdot \arcsin \left(\frac{s}{R \sin \theta_{z}}\right)
$$

The results of this correction are shown in Fig. 6b. It is easy to see that the separation between the points is more homogeneous over the section of the surface of the virtual sphere, which reduces the quantity of points and measure time needed for the angular response calculations.

Accurate measurements of the angular response require that the scanned area is centred on the optical fibre (see Fig. 7, position B), and that the distance to the optical fibre remains constant; yet, this increases the number of measured points. To reduce the number of measurements, we moved the lamp over the surface of a virtual sphere centred on the entrance optics. That way, the angle from the optical fibre $\theta$ is different from the angle $\alpha$ formed by the lamp position, the centre of the entrance optics and the optical fibre aperture. That is why we applied a correction of the viewed angle $\theta$ using Eq. 2.

$$
\theta=\alpha+\arctan \left(\frac{r \sin \alpha}{d+r(1-\cos \alpha)}\right)
$$

where $r$ is the radius of the entrance optics, $d$ is the distance from the lamp to the optical fibre at $\theta$ equal zero and $\alpha$ refers to the position of the lamp in the robot coordinate system. Owing to the correction applied to all measurements, a wider angular response was achieved.

\section{Optical properties of the $100 \mathrm{~W}$ lamp}

Since the lamp was moved tangentially to the surface of a virtual sphere, its irradiance changed. Therefore, we analysed the distribution of the emitted radiation of the lamp in the horizontal and vertical planes and its influence on the angular response measurements using the robotic setup.

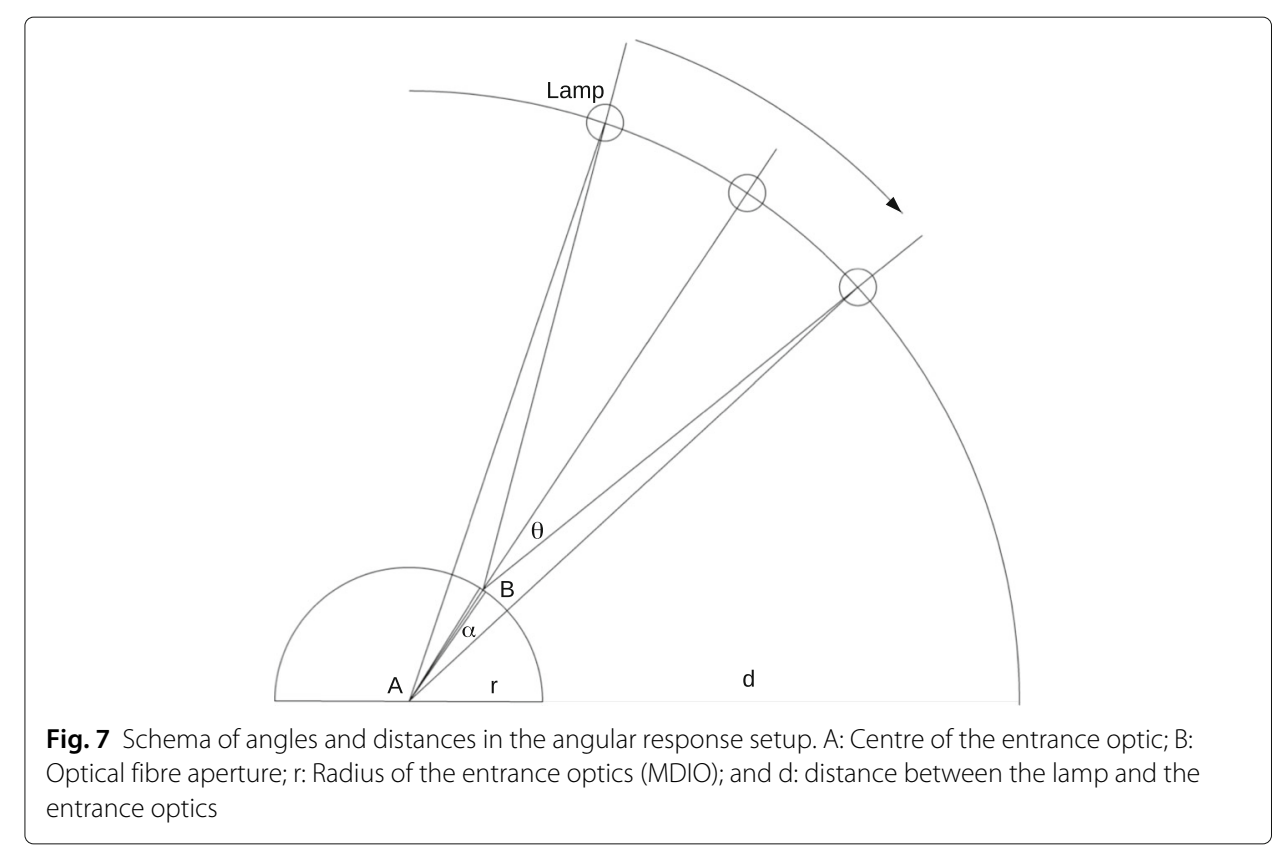




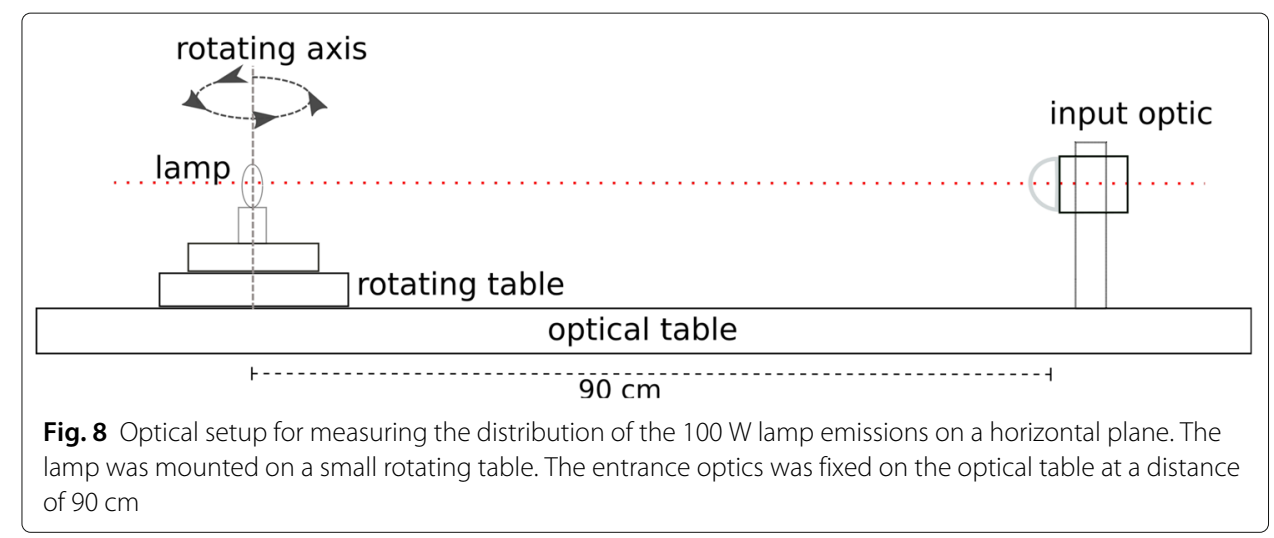

\section{Distribution of radiant exitance as a function of the horizontal rotation}

The experimental setup consisted of an $100 \mathrm{~W}$ low-voltage halogen lamp without reflector (OSRAM model $64623 \mathrm{HLX}$ ), which was mounted on a $0.25^{\circ}$ resolution rotating table (Fig. 8). To measure the emission of the lamp, a global irradiance entrance optics was mounted with a distance of $90 \mathrm{~cm}$ from the lamp on the optical table. For aligning the lamp with the input optics, a laser was used. The irradiance was measured by our NDACC-certified (Network for the Detection of Atmospheric Composition Change) double-monochromator spectroradiometer TMc300 Bentham Instruments, $300 \mathrm{~mm}$ focal length, and 2400 lines per mm grating with a spectral range of $250 \mathrm{~nm}$ to $2500 \mathrm{~nm}$ $[23,24]$. The spectroradiometer was connected to the irradiance entrance optics through an optical fibre and the lamp was manually rotated from $-110^{\circ}$ to $+100^{\circ}$ at angular intervals of $10^{\circ}$.

The maximum irradiance emitted by the lamp was measured at an angle of $0^{\circ}$ (see Fig. 9). Furthermore, the minimum irradiance was measured at $\pm 90^{\circ}$, corresponding to a value of $55 \%$ of the maximum irradiance. This discrepancy is due to the physical structure of the lamp, which consists of a metallic filament (i.e., a spiral with two wires connected

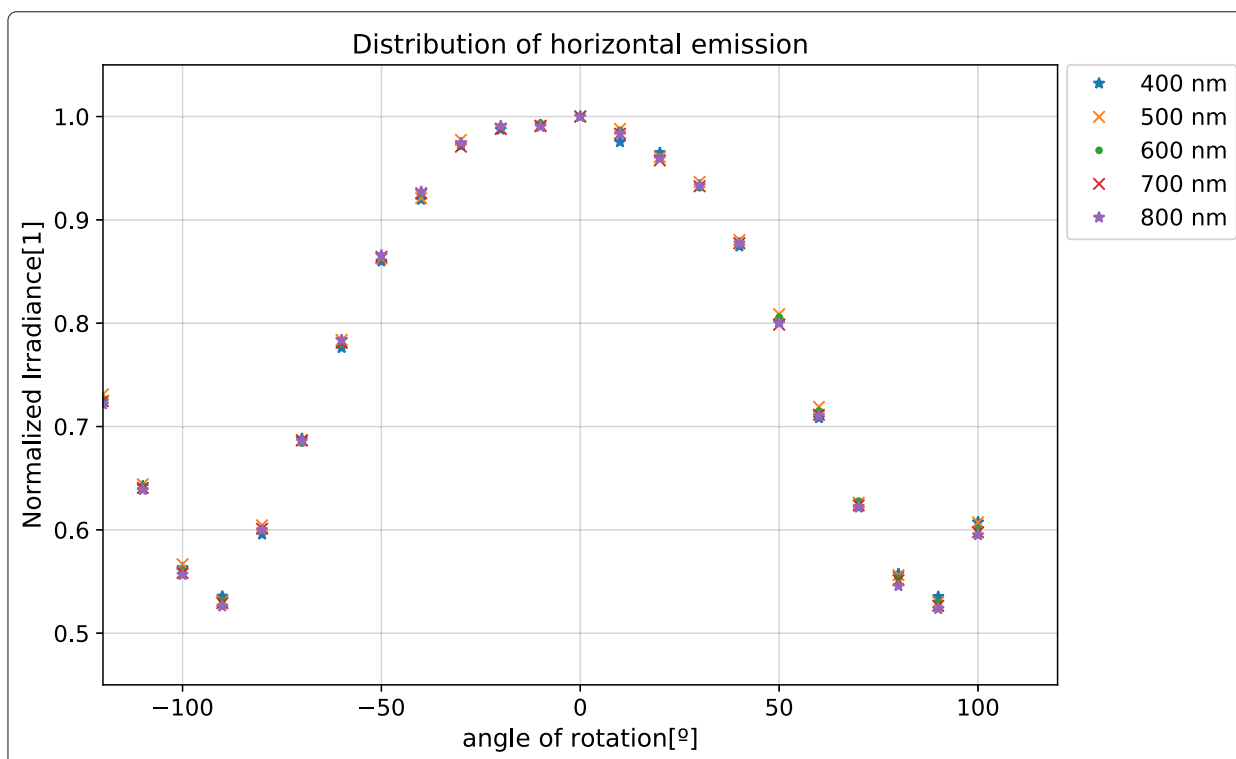

Fig. 9 Distribution of radiant exitance of the $100 \mathrm{~W}$ halogen lamp on the horizontal plane. The irradiance was measured at steps of $10^{\circ}$ 


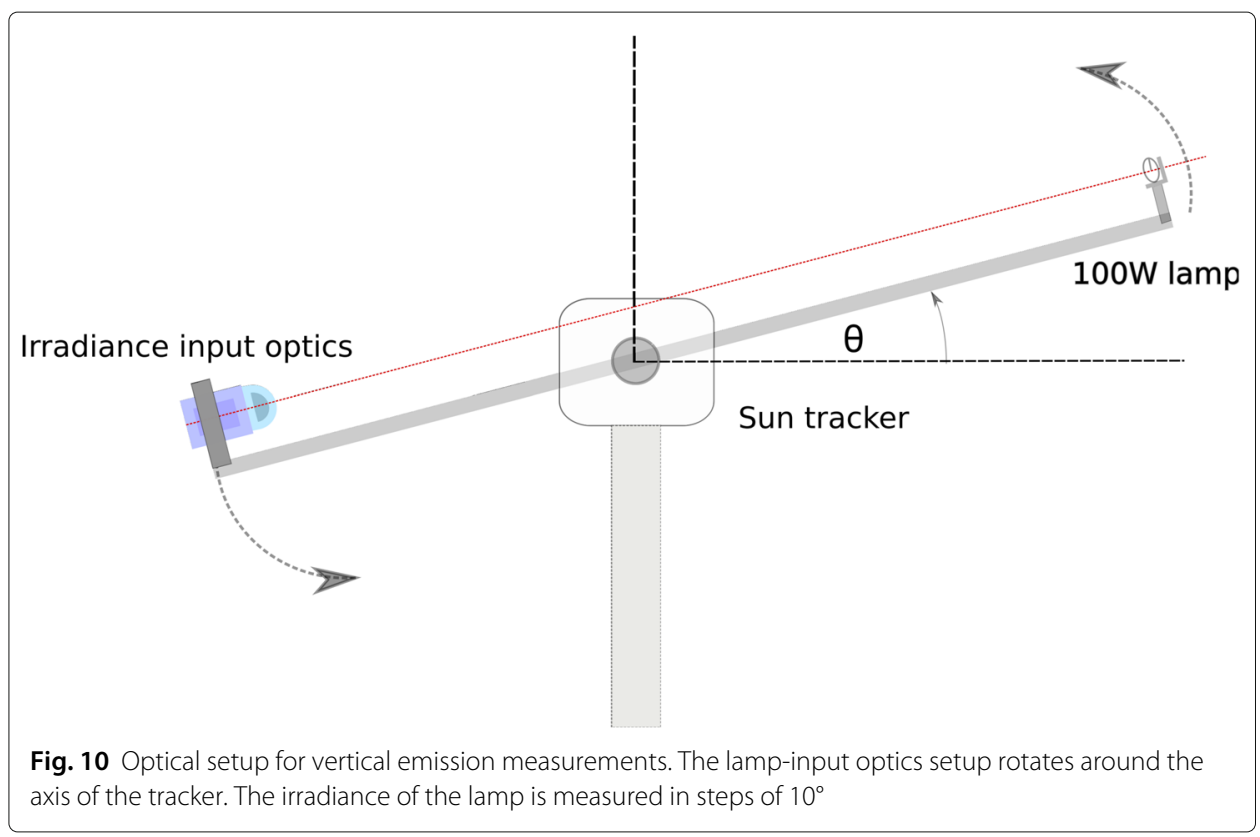

to the base). At an angle of $0^{\circ}$, the area of the filament facing the entrance optics was at its maximum $\left(12.69 \mathrm{~mm}^{2}\right)$. For an angle of $\pm 90^{\circ}$, the area of the filament was only $5.72 \mathrm{~mm}^{2}$. A clear wavelength dependence was not found. We can conclude that in order to prevent the effects in the change of irradiance, the lamp needs to point to the entrance optics with the same surface area.

\section{Distribution of radiant exitance as a function of the vertical rotation}

The measurements of the emissions in a vertical plane were carried out by using an ENEO positioning system with a pan/tilt head that included a heater. A metallic bar was mounted along the zenith axis of the tracker (Fig. 10). At one extreme, the same $100 \mathrm{~W}$ halogen lamp was fixed. At the other extreme, an irradiance entrance optics was mounted and aligned to the lamp. The lamp-entrance optics setup was rotated in the zenith axis at $10^{\circ}$

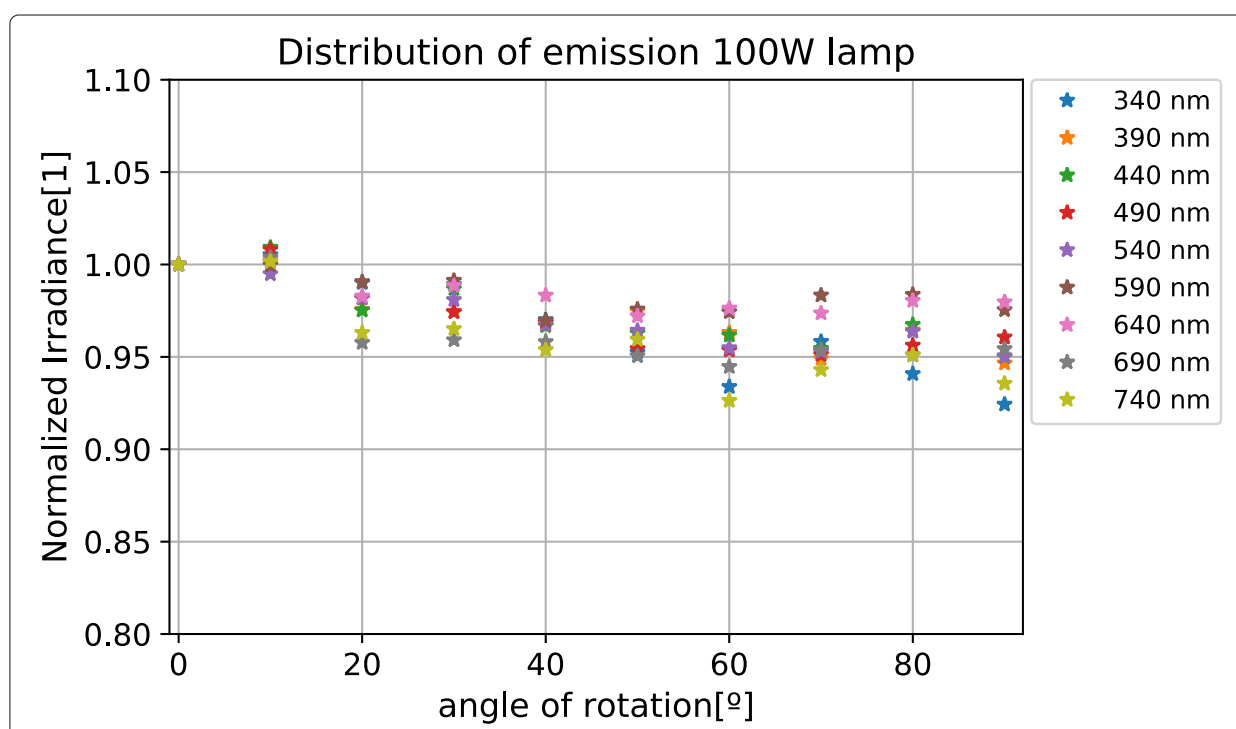

Fig. 11 Normalized irradiance measured in a distance of $90 \mathrm{~cm}$ from a $100 \mathrm{~W}$ lamp as function of the rotation angle in the zenith. The radiant exitance of the lamp decreases up to $7 \%$ when it is rotated $90^{\circ}$ 
intervals, ranging from $0^{\circ}$ to $90^{\circ}$. The distance between the lamp and the input optics was $90 \mathrm{~cm}$ during the measurement.

As shown in Fig. 11, the emission of the lamp changes with the rotation angle. For an angle of $\theta=0^{\circ}$ (lamp normal to the ground), the irradiance is at its maximum, while it decreases as a function of the rotating angle up to $8 \%$ at $90^{\circ}$.

Since the lamp was moved tangentially over the virtual sphere, the horizontal variation of the emission was not a problem. However, a correction of the magnitude of the measured irradiance was necessary for the vertical movement if the scanning range was larger than $30^{\circ}$.

\section{Results and discussion}

Angular response of an optical fibre

For a first approximation to the characterization of the input optics, the angular response of only one optical fibre was analysed. For this purpose, we used the optical fibre \# 4 located at $10.5^{\circ} \mathrm{ZA}$ (zenith angle), $180^{\circ}$ azimuth angle (AA). The robotic arm was programmed to perform an angular scan of $\pm 20^{\circ}$ in the azimuth and $\pm 10^{\circ}$ in the zenith directions, using the optical fibre position as the centre of the scanned area. For angles between $\pm 7^{\circ}$ with respect to the optical fibre position, the angular step was reduced to $0.5^{\circ}$; for the rest of the positions, an angular step of $1^{\circ}$ was chosen, resulting in a scan time of around 2 hours. Approximately 1500 different points were used to determine the two-dimensional angular response. Figure 12 shows the contour plot of the normalized radiance measured by the optical fibre \# 4 as function of the lamp position. White dots represent the position of the lamp over the virtual sphere during the measurements.

The centre of the angular response should be aligned with the intended observation angle (optical fibre). However, the shape of the angular response is nonsymmetric, with the highest variations found over values of 0.5 (yellow and red area in Fig. 12). This leads

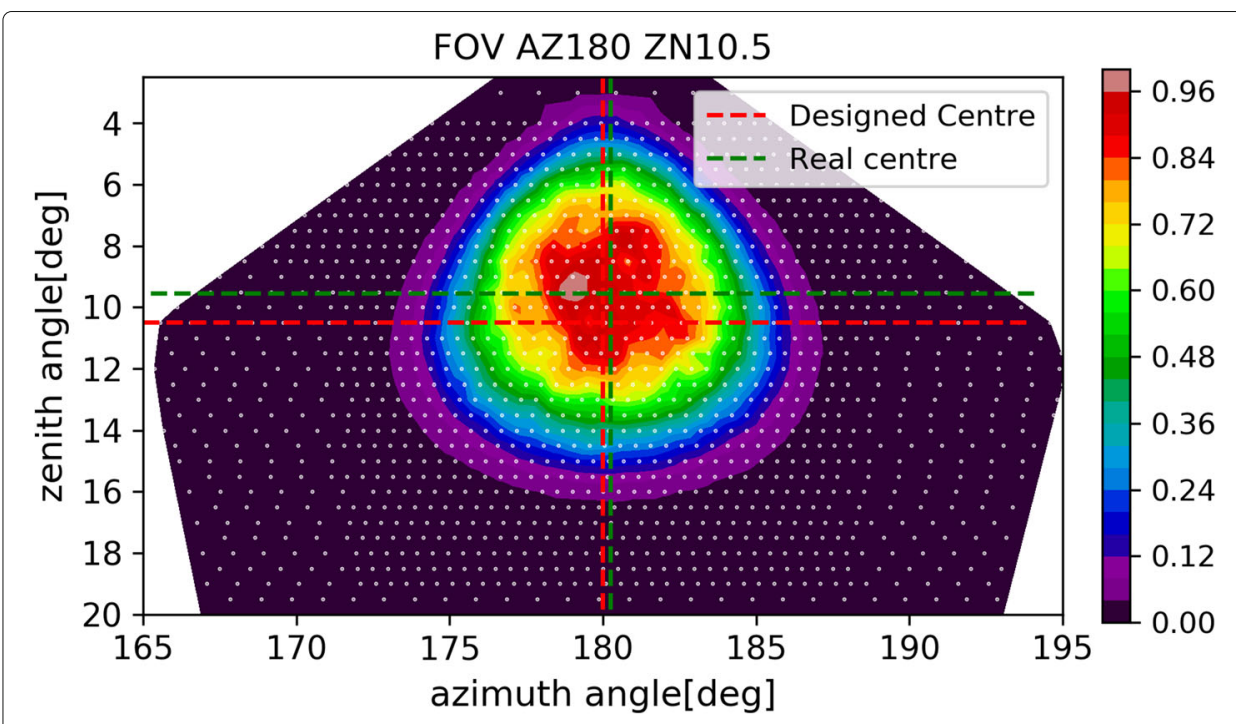

Fig. 12 Normalized radiance ( $644 \mathrm{~nm}$ wavelength) measured through the optical fibre located at $10.5^{\circ} \mathrm{ZN}$ and $180^{\circ} \mathrm{AA}$ as a function of the zenith and azimuth angles. White dots represent the position of the lamp over the virtual sphere during the measurement. The intersection of the dotted red lines indicates the expected angular position of the optical fibre. The intersection of the dotted green lines indicates its actual position 
to a displacement of the weighted maximum of the angular response. The field-of-view (FOV) of an optical system is often expressed as the maximum angular size of the object as seen from the entrance pupil [25], it can be calculated by the Full Width at Half Maximum (FWHM) of the angular response curve. A way to find the centre of the FOV is to use the centre-of-mass (MC), which in this case weighs the radiance as a function of position and wavelength. It is calculated by using Eq. 3

$$
M C(\lambda)=\frac{1}{\sum_{i=0}^{n} L_{i}\left(\lambda, \vec{r}_{i}\right)} \sum_{i=0}^{n} L_{i}\left(\lambda, \vec{r}_{i}\right) \cdot \vec{r}_{i}
$$

where $L$ is the spectral radiance, $\lambda$ is the wavelength and $r$ is the position of the lamp over the virtual sphere.

The calculation of the $\mathrm{MC}$ in the azimuth direction, resulted in the central FOV position ranged from $180.2^{\circ}$ to $180.6^{\circ}$ depending on the wavelength. Since the designed position of the optical fibre on AMUDIS is $180^{\circ} \mathrm{AA}$, the maximum shift amounts to $0.6^{\circ}$. The shift along the zenith was larger, ranging from $10^{\circ}$ at $434 \mathrm{~nm}$ to $9.5^{\circ}$ at $800 \mathrm{~nm}$. For the whole spectrum, the shift in position amounts to $0.5^{\circ}$. The average FOV central position of the whole spectrum, was estimated to be $180.19^{\circ}$ for the azimuth and $9.7^{\circ}$ for the zenith direction. For the integrated radiance, the shifts in weighted FOV central position were found to be only up to $0.19^{\circ}$ in the azimuth and up to $0.01^{\circ}$ in the zenith direction. Figure 13 shows the angular response along the azimuthal and zenithal directions; the red dots represent the measured radiance while the blue line is the smoothed radiance obtained by applying the Savitzky-Golay filter [26] to the spectral radiance data.

The FOV was calculated by: 1) interpolating the angular response on the scanned area; 2) selecting the azimuth and zenith values that cross the weighted centre of the FOV (green line in Figs. 13); and 3) plotting the radiance along the azimuth or zenith axis as a function of the angle. According to this procedure, the FOV values was $8.12 \pm 0.01^{\circ}$ for the azimuth axis (Fig. 13 a) and $8.14 \pm 0.01^{\circ}$ for the zenith axis (Fig. $13 \mathrm{~b}$ ). The results suggest that the FOV has a nearly circular shape since both values for zenith and azimuth are similar.

\section{Angular positions of the FOV of AMUDIS input optics}

Results in subsection "Angular Response of an Optical Fibre" suggest that it is only necessary to use a 1-degree angular step to measure the FOV of the entrance optics (which

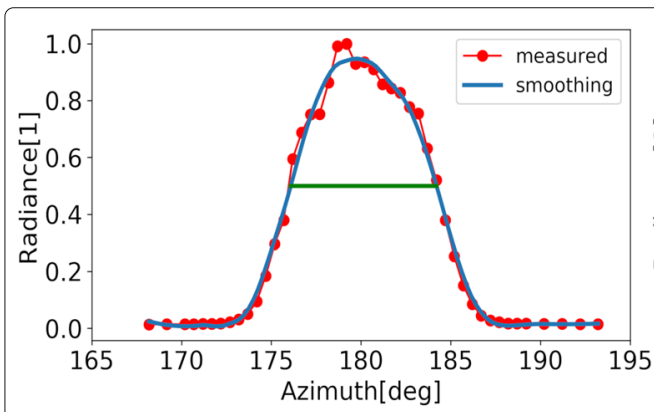

a)

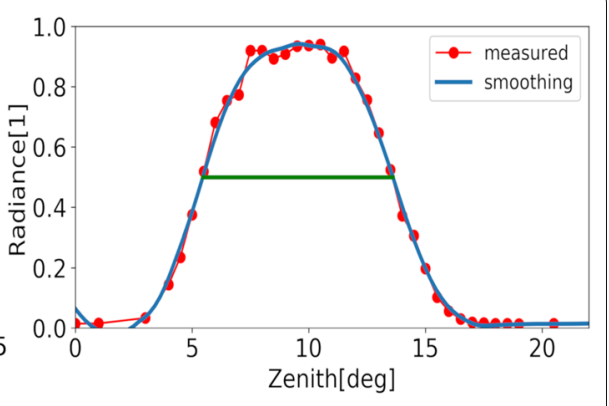

b)

Fig. 13 Angular response at $644 \mathrm{~nm}$ of the optical fibre \# 4 along the a) azimuth and b) zenith directions at $644 \mathrm{~nm}$. The green line stands for the FWHM of the smoothed curve 
results in a reduction of the measurement time). Figure 14 shows the results of performing the scan over the entire virtual sphere and applying the corrections described in section "Optimization of the method" 1. Figure 14 shows the designed/expected position of the optical fibres and the actual position at $502 \mathrm{~nm}$ and $830 \mathrm{~nm}$. As shown in Fig. 14, the actual positions of the FOV differ from the expected positions for most of the entrance optics. For example, for fibres \# 5, 61, 114, and 144, the actual position of the FOV centre are displaced by a few degrees with respect to the expected positions, whereas fibres \# 64, 72, and 103 were correctly aligned. We can conclude that the fibre misalignment are a characteristic of the entrance optics that must be corrected by a full characterization of the instrument.

In addition to detecting the FOV central position of the fibres, it is also possible to show the FOVs of a single wavelength in one figure, as shown in Fig. 15. The measured radiance at each point across the virtual surface was normalized and plotted for a wavelength of $644 \mathrm{~nm}$. As shown in Fig. 15, the FOVs are irregular both in shape and distribution. The blue area indicates where the FOV of the corresponding fibre is not recognized as the fibre was broken and does not transmit radiation into these sections. The signal measured by each optical fibre is different and therefore, the responsivity of the optical fibres is also different. In addition, it is possible to see some overlaps of the signal at the edge of the FOVs in the fibres \#133 and 134, or 137 and 138. A stronger overlap is shown in the fibre \#48. Some blue areas occur in the FOV of some fibres. This is due to overlapping of the measurements at these locations. It could be improved by using a new method to extract only the highest signal measured by one of both optical fibre.

\section{Conclusions}

In this paper we have developed a method for the characterization of a multi-directional entrance optics for sky radiance measurements fitted with 435 different optical fibres uniformly distributed along 145 directions. The experimental setup for the characterization consists of a robotic arm and a $100 \mathrm{~W}$ halogen lamp. The robot moves the lamp over

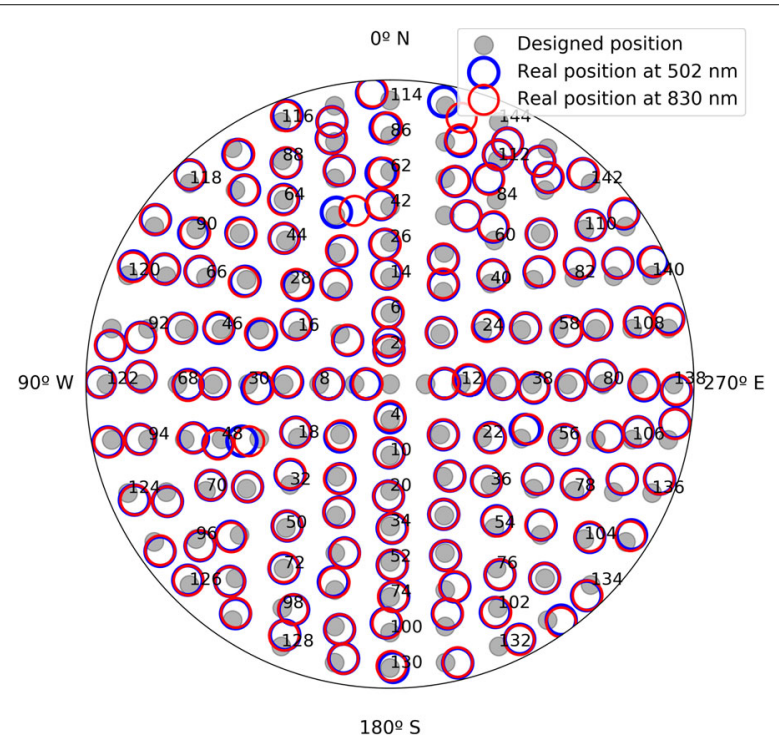

Fig. 14 Expected and actual FOV positions of the AMUDIS input optics. The red and blue circles represent the FOVs of the optical fibres at $502 \mathrm{~nm}$ and $830 \mathrm{~nm}$ respectively. The optical fibres are represented by grey circles 


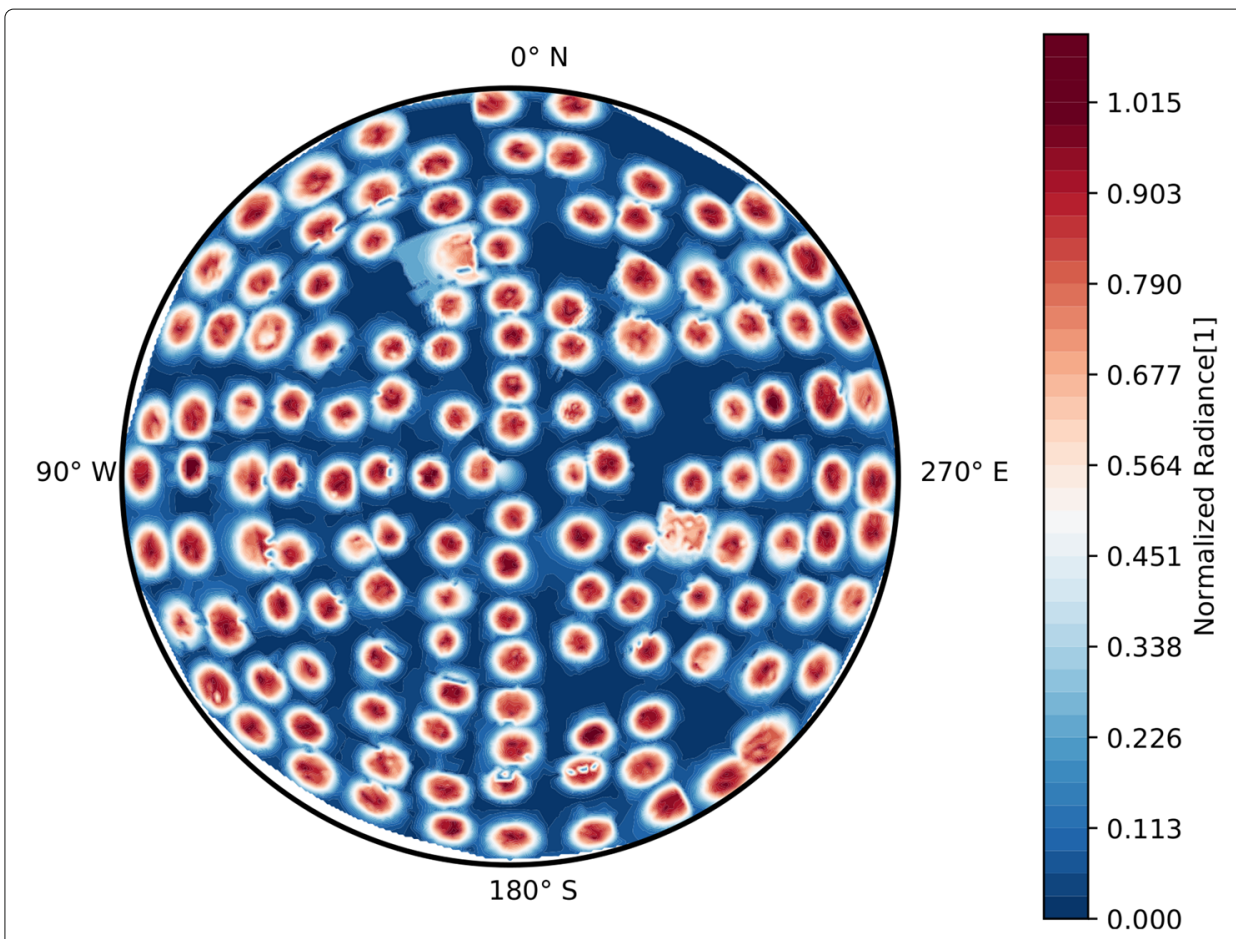

Fig. 15 Angular response of the AMUDIS input optics at $644 \mathrm{~nm}$. Figure obtained from 20.949 uniformly distributed measurements over a virtual sphere of radius $102.5 \mathrm{~cm}$ and centred on the AMUDIS entrance optics

the surface of a virtual sphere of $102.5 \mathrm{~cm}$ radius around the entrance optics. Measurements of the radiance emitted by the lamp from different positions over the virtual sphere, allowed in the characterization of the FOVs of the multi-directional entrance optics. The characterization revealed the actual FOV and the direction of observation of the sky for each of the fibres on the entrance optics.

Our results suggest that there are still opportunities for improving the development of the entrance optics as the angular response of the entrance optics exhibited some misalignments. These misalignments are likely attributable to the manufacturing process of the entrance optics. A possible cause could be that the cut of the optical fibres is not perfect (the diameter is very small $(50 \mu \mathrm{m}))$. Misalignments could also arrive from during the bonding of the fibre to the aluminium dome. Finally, the shape of the FOV is not circular, which results in different angular responses of the optical fibres. Nevertheless note that the misalignments of the optical fibres remains small compared to the size of the FOV (up to $15 \%$ of the FOV size). It is also important to note that FOV values range from $12^{\circ}$ at $400 \mathrm{~nm}$ to $5^{\circ}$ at $880 \mathrm{~nm}$. Despite the misalignment, there are only four fibres whose FOV overlaps at shorter wavelengths. For wavelength greater than $550 \mathrm{~nm}$, there is no more overlapping.

The new characterization method allowed measuring the FOV of multi-directional input optics with a high spatial resolution (up to $0.5^{\circ}$ ). This new method is expected to significantly improve the quality of the spectral radiance measurements, allowing retrieving the shape and direction of the FOV of each optical fibre of the entrance optics with high accuracy. 


\begin{abstract}
Abbreviations
AA: Azimuth angle; AMUDIS: Advanced multidirectional spectroradiometer; CCD: Charge-coupled device; FOV: Field of view; FWHM: Full width at half maximum; HSI: Hemispherical Sky Images; MC: Centre of mass; MDIO: Multidirectional input optics; MUDIS: Multidirectional spectroradiometer; NDACC: Network for the detection of atmospheric composition change; NIR: Near infrared; UV: Ultraviolet; VIS. Visible; ZA: Zenith angle
\end{abstract}

\title{
Acknowledgements
}

Not applicable.

\section{Authors' contributions}

EL contributed in writing of the manuscript. AN and JD helped with the measurements in the laboratory and technical support. GS contributed to the development and ideas for the experimental setup. MT wrote most of the manuscript with input from all authors, analysed the datasets and implemented the experimental setup. All authors read and approved the final manuscript.

\section{Authors' information}

Not applicable.

\section{Funding}

This investigation was founded by the DAAD-CONICYT scholarship, supporting the maintenance of the first author during the research time. The Institute of Meteorology and Climatology of the Leibniz University of Hannover, Germany, by the acquisition of the robotic arm and providing the laboratory of Solar Radiation. AMUDIS has been funded by DFG INST 187/555-1 FUGG. Open Access funding enabled and organized by Projekt DEAL.

\section{Availability of data and materials}

The datasets used and/or analysed during the current study are available from the corresponding author upon request.

\section{Declarations}

\section{Competing interests}

The authors declare that they have no competing interests.

Received: 22 December 2020 Accepted: 24 June 2021

Published online: 28 July 2021

\section{References}

1. Fröhlich C, Brusa R (1981) Solar radiation and its variation in time. Sol Phys 74(1):209-15. https://doi.org/10.1007/ BF00151291

2. Cordero R, Damiani A, Da Silva L, Laroze D, Labbe F (2013) Spectral UV radiance measured at a coastal site: A case study. Photochem Photobiol Sci 12(7):1193-201. https://doi.org/10.1039/C3PP25440B

3. Cordero R, Damiani A, Ferrer J, Rayas J, Jorquera J, Tobar M, Labbe F, Laroze D (2013) Downwelling and upwelling radiance distributions sampled under cloudless conditions in Antarctica. Appl Opt 52(25):6287. https://doi.org/10. 1364/AO.52.006287

4. Pissulla D, Seckmeyer G, Cordero R, Blumthaler M, Schallhart B, Webb A, Kift R, Smedley A, Bais A, Kouremeti N, Cede A, Herman J, Kowalewski M (2009) Comparison of atmospheric spectral radiance measurements from five independently calibrated systems. Photochem Photobiol Sci Off J Eur Photochem Assoc Eur Soc Photobiol 8(4):516-27

5. Riechelmann S, Schrempf M, Seckmeyer G (2013) Simultaneous measurement of spectral sky radiance by a non-scanning multidirectional spectroradiometer (MUDIS). Meas Sci Technol 24(12):125501

6. Kouremeti N, Bais A, Kazadzis S, Blumthaler M, Schmitt R (2008) Charge-coupled device spectrograph for direct solar irradiance and sky radiance measurements. Appl Opt 47:1594-607. https://doi.org/10.1364/AO.47.001594

7. Wuttke S, Seckmeyer G (2006) Spectral radiance and sky luminance in Antarctica: A case study. Theor Appl Climatol 85(3-4):131-48. https://doi.org/10.1007/s00704-005-0188-2

8. Blumthaler M, Gröbner J, Huber M, Ambach W (1996) Measuring spectral and spatial variations of UVA and UVB sky radiance. Geophys Res Lett 23(5):547-50. https://doi.org/10.1029/96GL00248

9. Kuchinke C, Fienberg K, Nunez M (2004) The Angular Distribution of UV-B Sky Radiance under Cloudy Conditions: A Comparison of Measurements and Radiative Transfer Calculations Using a Fractal Cloud Model. J Appl Meteorol 43(5):751-61. https://doi.org/10.1175/2095.1

10. Bruegge C, Helmlinger M, Conel J, Gaitley B, Abdou W (2000) PARABOLA III: A sphere-scanning radiometer for field determination of surface anisotropic reflectance functions. Remote Sens Rev 19(1-4):75-94. https://doi.org/10.1080/ 02757250009532411

11. Bruegge C, Coburn C, Elmes A, Helmlinger M, Kataoka F, Kuester M, Kuze A, Ochoa T, Schaaf C, Shiomi K, Schwandner F (2019) Bi-Directional Reflectance Factor Determination of the Railroad Valley Playa. Remote Sens 11(22):2601. https://doi.org/10.3390/rs11222601

12. Deering D (1988) PARABOLA Directional Field Radiometer For Aiding In Space Sensor Data Interpretations. In: Recent Advances in Sensors, Radiometry, and Data Processing For Remote Sensing. International Society for Optics and Photonics, Bellingham Vol. 0924. pp 249-261. https://doi.org/10.1117/12.945693

13. Román R, Antón M, Cazorla A, de Miguel A, Olmo F, Bilbao J, Alados-Arboledas L (2012) Calibration of an all-sky camera for obtaining sky radiance at three wavelengths. Atmos Meas Tech 5(8):2013-24. https://doi.org/10.5194/ amt-5-2013-2012 
14. Tohsing K, Schrempf M, Riechelmann S, Schilke H, Seckmeyer G (2013) Measuring high-resolution sky luminance distributions with a CCD camera. Appl Opt 52(8):1564. https://doi.org/10.1364/AO.52.001564

15. Zuber R, Stührmann A, Gugg-Helminger A, Seckmeyer G (2017) Technology for detecting spectral radiance by a snapshot multi-imaging spectroradiometer. Meas Sci Technol 28(12):125903. https://doi.org/10.1088/1361-6501/ aa9409

16. Seckmeyer G, Lagos Rivas L, Gaetani C (2018) Strahlungsbilanzen, Biological and Medical Effects of Solar Radiation. In: Promet, vol. Heft 100 (2018). Deutscher Wetterdienst, Fachinformationsstelle und Deutsche Meteorologische Bibliothek, Offenbach/M. p 119

17. Rettberg P (1999) Biological UV Dosimetry: A Tool for Assessing the Impact of UV Radiation on Health Ecosystems. Luxembourg EUR 19098 EN:68-75

18. Niedzwiedz A, Duffert J, Tobar M, Quadflieg E, Seckmeyer G (2021) Laboratory calibration for multidirectional spectroradiometers. Meas Sci Technol 32:9. https://doi.org/10.1088/1361-6501/abeb93

19. Seckmeyer G, Bais A, Bernhard G, Blumthaler M, Booth C, Disterhoft P, Eriksen P, McKenzie R, Miyauchi M, Roy C (2001) Instruments to measure solar ultraviolet irradiance. Part 1: Spectral instruments. In: WMO-GAW. WMO, Geneva. p 17

20. Knoop M, Diakite A, Weber N, Luo T, Abdelmageed A-E (2018) Spatially resolved spectral Sky Daylight Measurement Data: Methods of Collection, Evaluation and Representation. In: Proceedings of the Conference at the CIE Midterm Meeting 201723 - 25 October 2017, JEJU, Republic of Korea. International Commission on Illumination, CIE, Jeju Island, Republic of Korea. pp 1036-1046. https://doi.org/10.25039/x44.2017.PO68

21. Bernhard G, Seckmeyer G (1999) Uncertainty of measurements of spectral solar UV irradiance. J Geophys Res Atmos 104(D12):14321-45. https://doi.org/10.1029/1999JD900180

22. Universal Robots (2019) Technical specifications UR10. Universal Robots, USA. https://www.universal-robots.com/ media/50880/ur10_bz.pdf. Accessed 04 Nov 2019. https://doi.org/10.1117/3.547461

23. Wuttke S, Seckmeyer G, Bernhard G, Ehramjian J, McKenzie R, Johnston P, O'Neill M (2006) New Spectroradiometers Complying with the NDSC Standards. J Atmos Ocean Technol 23(2):241-51. https://doi.org/10.1175/JTECH1826.1

24. Cordero R, Seckmeyer G, Damiani A, Riechelmann S, Rayas J, Labbe F, Laroze D (2013) The world's highest levels of surface UV. Photochem Photobiol Sci 13(1):70-81. https://doi.org/10.1039/C3PP50221 J

25. Greivenkamp J (2004) Field Guide to Geometrical Optics. SPIE Press, Bellingham, WA

26. Persson P-O, Strang G (2003) Smoothing by Savitzky-Golay and Legendre Filters. In: Arnold D, Santosa F, Rosenthal J, Gilliam D (eds). Mathematical Systems Theory in Biology, Communications, Computation, and Finance. Springer New York, New York, NY Vol. 134. pp 301-15

\section{Publisher's Note}

Springer Nature remains neutral with regard to jurisdictional claims in published maps and institutional affiliations.

\section{Submit your manuscript to a SpringerOpen ${ }^{\circ}$ journal and benefit from:}

- Convenient online submission

- Rigorous peer review

- Open access: articles freely available online

- High visibility within the field

- Retaining the copyright to your article

Submit your next manuscript at $\boldsymbol{\triangleright}$ springeropen.com 